\title{
Effect of pH-11 Buffer as Agent Reaction Moderator in the Growth and Characterization of CdS Thin Films by CBD Technique
}

\author{
F. Vázquez Monroy ${ }^{1}$, A. García-Barrientos ${ }^{2}$, and R. Ramírez Bon ${ }^{3}$, J.L. Bernal ${ }^{2}$
}

1. Universidad Tecnológica Tula-Tepeji, Av. Universidad tecnológica No. 1000, col El 61, El Carmen, Tula de Allende Hidalgo, CP 42830, México

2. Universidad Politécnica de Pachuca, Carretera Pachuca-Cd. Sahagún, km 20, Ex-Hacienda de Santa Bárbara Municipio de Zempoala, Hidalgo, CP 43830, México

3. CINVESTAV-IPN Unidad Querétaro, Libramiento Norponiente 2000, Fracc. Real de Juriquilla, CP 76230, Querétaro, Qro. México

The effect of deposition parameters of CdS thin films developed by Chemical Bath Deposition (CBD) technique was investigated in this paper. We studied the influence of $\mathrm{pH}$ control of the reaction solution on the structural and optical properties of chemically deposited CdS films. Different films thicknesses of $\mathrm{CdS}$ were deposited onto a glass substrate. The structural surface morphology of as-deposited CdS thin films was characterized by SEM, XRD, profilometer, and ultraviolet-visible spectroscopy. The physical conditions were kept identical while growing the samples. The investigation of the effect of the synthesis method on the change the ammonium hydroxide by buffer $\mathrm{pH}-11$ contributed in increases the growth kinetics, resulting in thicker films.

Over the years, thin films of II-VI semiconductor compounds are most promising for utilization in solar cells, out of which the cadmium sulfide $(\mathrm{CdS})$ has received intensive attention, since its band gap lie very close to the range of maximum theoretically attainable energy conversion efficiency. CdS is an inorganic compound of yellow solid color, and due to its wide band gap, photoconductivity, and high electron affinity, is known to be an excellent heterojunction partner for p-type cadmium telluride, p-type copper indium diselenide. It has been widely used as a window material in high efficiency thin film solar cells based on cadmium telluride or copper indium diselenide [1-3]. In these devices, light penetrates the CdS layer and it is absorbed in the p-type semiconductor close to the $p n$ junction. Also, $\mathrm{CdS}$ is an interesting crystal material in the area of photodetectors, semiconductor lasers, and non linear integrated optical devices. The importance of wide band gap materials is related to the possibility of fabricating light emitting diodes or laser heterostructures for emission in the visible spectral range.

The CdS thin films were fabricated by CBD technique on glass substrate. The diffractogram of an as-deposited CBD-CdS sample is shown in figure 1(a). The XRD pattern shows only one line that corresponds to the reflection the mixture the (002) of the Greenockita (hexagonal) and (111) of the Hawleyite (cubic) peak (1), showing in general that the preferential orientation of the film is along the (002) and (111) direction others XRD pattern shows corresponds to the reflection of (220) of the hawleyite (cubic) peak (2) and (311) of the hawleyite (cubic) peak (3). However, we can observe also that the films have some amorphous component. The SEM photos (see figure 1(b)) show a surface of some porous homogeneous film having a grain size between 107 to $125 \mathrm{~nm}$ as shown in figure 1(c). In figure 1(d), the transmission spectrum of a CBD-CdS sample is presented; we can note a sharp absorption edge at about $275 \mathrm{~nm}$ (dashed line) which corresponds to a band gap of approximately 2.43 $\mathrm{eV}$. The transmittance at the high-energy region extends up to $300 \mathrm{~nm}$ as shown in the figure $1(\mathrm{~d})$. The film thickness was $66 \mathrm{~nm}$ and the resistivity of $1.17 \times 105 \Omega$-cm, this is an evidence of disorder effects or presence of amorphous components in the film. The use of the buffer also increases the growth kinetics, 
resulting in thicker films. However, the optical properties, such as energy band gaps at the critical points of the electronic band structure of $\mathrm{CdS}$, are not affected by the use of the buffer.

\section{References:}

[1] A. Mondal, et al., Solar Energy Materials, vol. 7, 431-438 pp., 1983.

[2] M.B. Ortuño-López, et al., Materials Research, vol. 16, 937-943 pp., 2013

[3] Kariper A, et al., Materials Chemistry and Physics, vol.129:183-188 pp., 2011.

[4] Mendoza-Pérez R., et al., Solar Energy, vol. 80, 682-686 pp., 2006

[5] Arreola-Jardón G., et al., Thin Solid Films, vol. 519, 517-520 pp., 2010.

[6] Mugdur PH, et al., Microscopy and Microanalysis. Vol.11,1954-5, pp. 2005

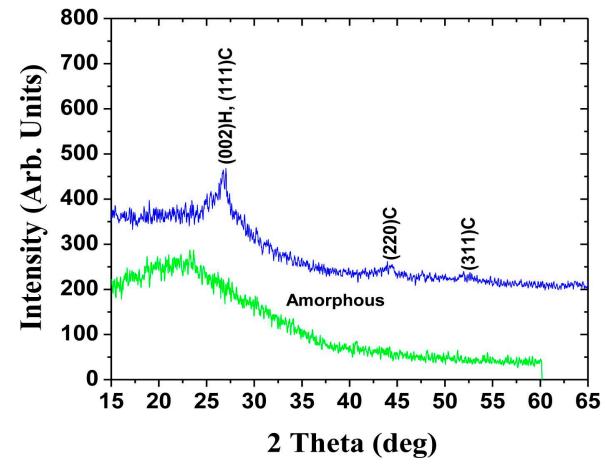

(a)

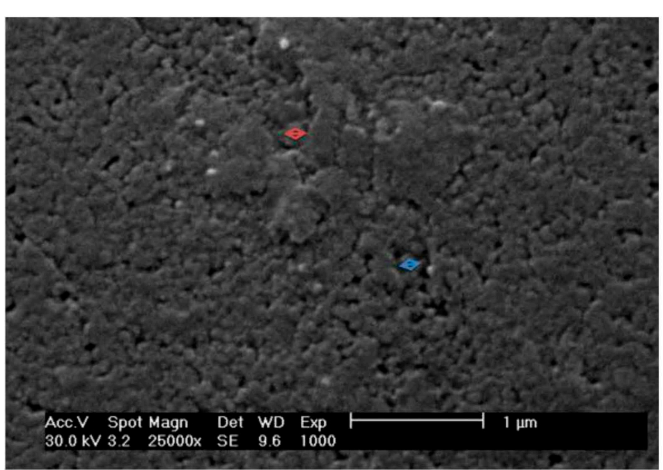

(c)

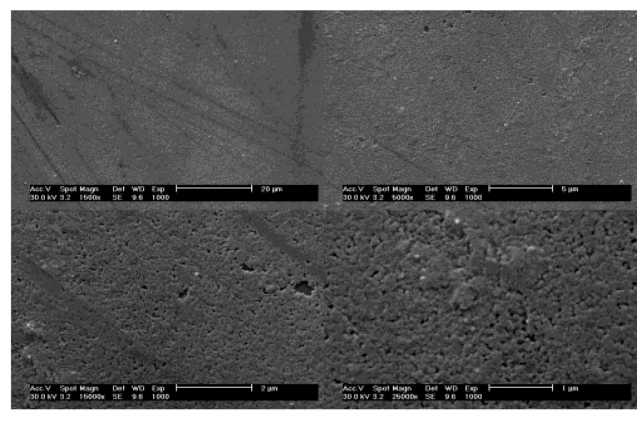

(b)

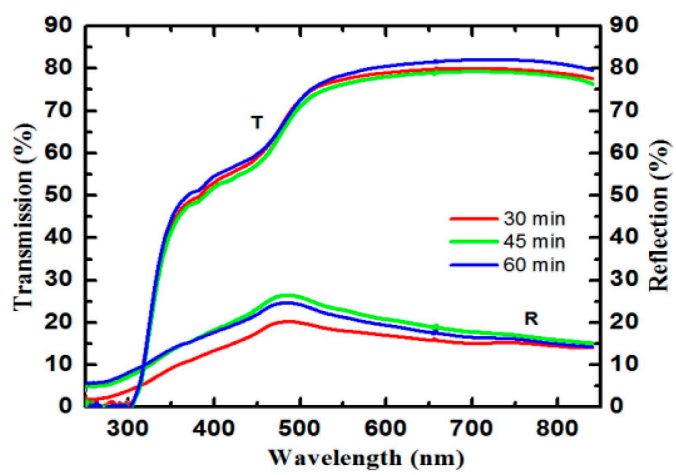

(d)

Figure 1. X-ray spectrum of a typical CBD-CdS sample (a). SEM photos of the sample photos having a grain size between $107 \mathrm{~nm}$ to $125 \mathrm{~nm}$ (b), (c). The transmittance at the high-energy extends up $300 \mathrm{~nm}$ (d). 